\title{
An Ontology Model-based Minnesota Code
}

\author{
Norbert Sram, Márta Takács \\ Óbuda University \\ Bécsi út 96/b, H-1034 Budapest, Hungary \\ E-mail: sramm.norbert@phd.uni-obuda.hu; takacs.marta@nik.uni-obuda.hu
}

\begin{abstract}
In this paper, the authors present an approach towards modeling a classical expert system using an ontology-based solution. The aim was to have an extensible setup, where multiple reasoning methods can be used, to provide the desired outcome. The case study, is a hierarchical rule-based system, for the evaluation of reference ECG signals called the Minnesota Code. This paper describes the practical limitations of the original expert system based definition, of the Minnesota code and describes an approach to represent it as an ontology that provides support for various reasoning methods. The authors present here, a possible solution to use the ontology model and ontology reasoning to provide a diagnostic evaluation of ECG information added to the Minnesota code ontology that corresponds to the rules defined by the expert system based solution.
\end{abstract}

Keywords: ontology; expert system; Minnesota Code

\section{Introduction}

Cardiovascular diseases are some of the most common causes of death. Based on the World Health Organization reports [9], about 30\% of deaths are caused by either Ischemic Heart Disease or stroke. The prediction of sudden cardiac deaths, is still a concern and mostly unsolved [6,7]. It is now well-recognized that classifications based on clinical circumstances can be misleading and often impossible, because $40 \%$ of sudden deaths may be unwitnessed [8]. There are examples of systems providing medical assistance to experts regarding diagnostics, using different models, based on expert systems and extended with novel mathematical models [16]. One of the authors has been involved in a research project which aims to shed further light on these cases by introducing telemedicine systems to alleviate the situation [10]. The authors of the paper suggest taking this one step further and introduce a proven diagnostic classification system on top of the monitoring system which could identify possible cardiovascular diseases. 


\section{The Minnesota Code}

The diagnostic classification system chosen by the authors is the Minnesota Code algorithm. The Minnesota Code [5] is a classification system for the electrocardiogram that utilizes a defined set of measurement rules to assign specific numerical codes according to severity of the ECG (Electrocardiography) findings. It is the most widely used ECG classification system in the world for clinical trials and epidemiological studies. It incorporates ECG classification criteria that have been validated, widely employed, and accepted by clinicians. From the definition's point of view, the Minnesota Code is a structured list of rules that examines certain characteristics of ECG waveforms. The Minnesota Code combines three major elements: a set of measurement rules, a classification system for reporting ECG findings and a set of exclusion rules. The relationship between the three major sets is vaguely defined.

\subsection{General Overview of the Diagnostic System}

In order to be able to provide a complete diagnostic result with the Minnesota code, it is required to have a 12-lead ECG and the corresponding various parameters of the ECG. This means approximately 55 parameters for each lead. In practice, providing all these parameters can pose significant problems. This is one of the weaknesses of the Minnesota code. Because of the numerous input parameters, the dependencies between various diagnostic rules, the rigid value definition used by the diagnostic rules, the results of the Minnesota code are sensitive to the precision of the input information. By introducing partial processing and measurement error toleration the usability of the diagnostic system could be greatly improved. In the case of an insufficient input dataset, the diagnostic system can still fall back to partial processing. In addition, measurement errors need to be taken into consideration.

The Minnesota code organizes the diagnostic rules based on ECG leads and waveform types. Each diagnostic rule has a unique identification (for example 1.1.1) and in some cases they are referred by multiple ECG lead groups. To resolve a diagnostic rule, it is required to take into consideration all occurrences of that rule. The output of a diagnostic rule equals the aggregation of the results of all evaluated occurrences of the specific rule. The Minnesota code definition states that the aggregation is done with the logical 'and' operator.

\subsection{Practical Usage of the Diagnostic System}

The input of the diagnostic system is an ECG cycle (heartbeat cardiac cycle) and its corresponding waveforms (P, Q, R, S, T) for all the 12 ECG leads (I, II, III, V1, V2, V3, V4, V5, V6, aVR, aVL, aVF). The waveforms are the visually 
identifiable parts of the ECG signal, that are used to characterize the ECG signal based on the waveform properties such as duration, amplitude, etc. The process of identifying the waveforms in a cardiac cycle is called annotation. The annotation of the signal can be done by hand or in an automated way using various algorithms [15]. Figure 1 displays a single ECG cycle with its corresponding waveforms annotated. From the point of evaluating the diagnostic rules, this is not relevant. The Minnesota Code deduces the diagnostic output based on annotated ECG signals. In practice this means that the recorded ECG signal is coupled with the required annotation values and this composition is forwarded to the diagnostic system. The output of the system is the state of the Minnesota codes for the examined ECG input. In detail, this means that the diagnostic system examines whether an input ECG cycle and annotations meet the requirement of one or more diagnostic rules (for example the rule 1.1.1). Since the goal is to provide partial diagnostic support besides providing the "true" and "false" states, the missing rule states convey information as well. If a diagnostic rule code is not present in the output, it means that the required information for evaluating that diagnostic rule was not available.

The length of an ECG recording varies in practice, from a few seconds up to several hours. In the case of the ECG databases used herein, the length of the recording varies. In order to avoid the redundant and extreme cases, the ECG recordings are preprocessed before the diagnostic steps. The preprocessing covers the identification of the "typical beats" for ECG records that contain multiple full ECG cycles. The output of the preprocessing for these cases is 1-3 ECG cycles (Figure 1, displays a single cycle) which correspond to the "average" ECG samples of the recording.

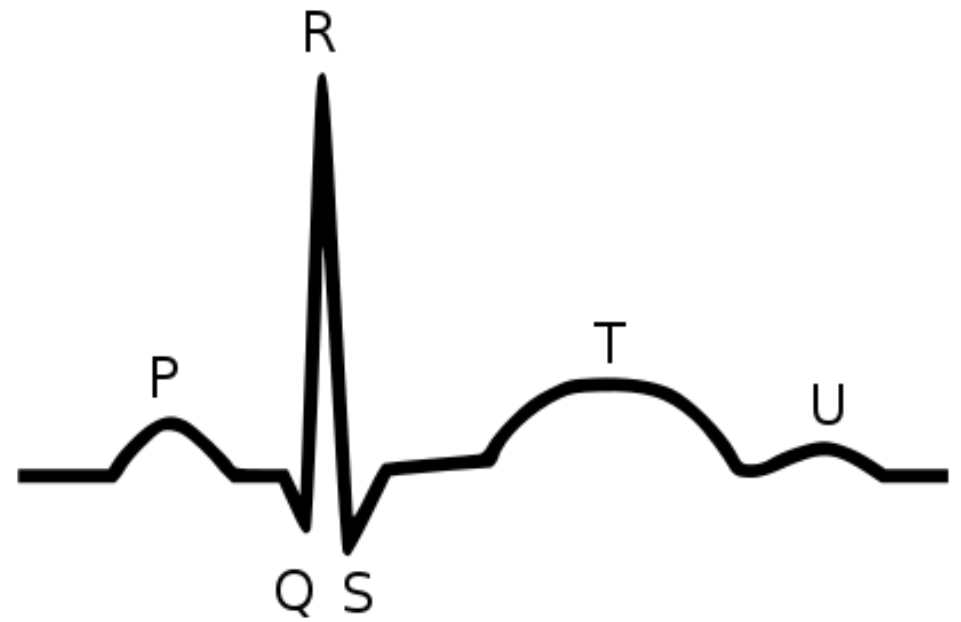

Figure 1

A cardiac cycle (heartbeat) with the corresponding waveforms highlighted on the signal [12] 
Measurement rules can have various properties and definitions, but most follow a common format. As an example, the diagnostic rule identified as 1-1-1 has the following definition and is made up of multiple statements (one for each ECG lead group):

$Q / R$ amplitude ratio $\geq 1 / 3$ and $Q$ duration $\geq 0.03$ sec in lead I or V6

$Q / R$ amplitude ratio $\geq 1 / 3$ and $Q$ duration $\geq 0.03$ sec in lead II

$Q / R$ amplitude ratio $\geq 1 / 3$ and $Q$ duration $\geq 0.03 \mathrm{sec}$ in any lead V2, V3, V4, V5

The rule 1-1-1 is true if one of the statements is fulfilled.

The exclusion rules define which diagnostic rules need to be ignored once a specific exclusion rule's requirements are met. Not all diagnostic rules can be ignored through exclusion rules. The Minnesota Code [5] classification system defines a table that contains the exclusion rules. Table 1, displays a subset of the exclusion rule definitions.

Table 1

Example of the Minnesota code incompatible rule definitions

\begin{tabular}{|l|l|}
\hline Code & Suppress this code(s) \\
\hline All $Q$-, QS-codes & $7-6$ \\
\hline$Q>0.03$ in lead I & $7-7$ \\
\hline $3-1$ & $1-3-2$ \\
\hline $6-8$ & All other codes \\
\hline
\end{tabular}

The classification is done through diagnostic rules, by identifying baseline ECGs that are used to categorize a study population into groups based on major and minor abnormalities. The classification definitions are similar to the exclusion rule definitions in the sense that each ECG baseline is associated with a set of diagnostic rules. Table 2 shows an entry of the categorization definitions.

Table 2

Classification code for prevalent ECG abnormalities

\begin{tabular}{|l|l|l|}
\hline \multicolumn{3}{|l|}{ ECG Categories Associated With Myocardial Infarction / Ischemia } \\
\hline Definition and Description & $\begin{array}{l}Q \text { wave MI; major Q waves } \\
\text { with or without ST-T } \\
\text { abnormalities }\end{array}$ & M-1-x \\
\hline Q wave MI & $\begin{array}{l}Q \text { wave MI; moderate Q } \\
\text { waves with ST } \\
\text { abnormalities }\end{array}$ & $1-1-1$ plus 4.1, 4.2 \\
\hline $\begin{array}{l}\text { Isolated minor Q and ST- } T \\
\text { abnormalities }\end{array}$ & $\begin{array}{l}\text { Minor Q waves without ST - } \\
\text { Tabnormalities }\end{array}$ & $1-3-x$ \\
\cline { 2 - 3 } & Minor ST-T abnormalities & $4-3,4-4,5-3,5-4$ \\
\hline
\end{tabular}


The classification table is used to provide the diagnostic output. As Table 2 shows, the classification table is grouped based on ECG categories, where each row is a specific entry. The Minnesota Code column of Table $2 \mathrm{~F}$ contains the requirements for the entries. Based on this, it is possible to produce a diagnostic result using the diagnostic rules that met their requirements.

Several studies have shown that the effectiveness of the computer-based Minnesota Code evaluation of ECG signal can be as effective as humans are with visual analysis [1]. There were studies that showed that the precision of the Minnesota Code system can be improved through the application of Fuzzy Logic [2]. These facts led to the decision to choose the Minnesota Code as the knowledge base.

Based on previous experiences gained in the modification and improvements of the Minnesota Code, the diagnostic rule set does not address the case of missing or incomplete inputs which, in turn, leads to insufficient diagnostic results for practical cases [4]. In order to incorporate improvements into the whole diagnostic process and not just a specific group [2], the definition and structure of the Minnesota Code needs to be more robust. There are efforts for providing a coherent and robust system for medical applications and providing a semantic representation of the patient data [11]. The Minnesota code-related research has not yet addressed the topic of semantically defining the diagnostic rules and dependencies. In order to establish a robust and extensible representation of the Minnesota Code-based diagnostic process, the authors needed to define a suitable representation. For this, they chose to follow the existing, general principles of semantic medical coding systems [11] and create an Ontology model for the Minnesota Code, which is described in detail in this paper.

\section{Ontology}

In order to restructure the representation of the Minnesota Code, there are multiple requirements that need to be taken into consideration, that are not provided by the current definition and its structured representation:

- The possibility to clearly identify the relationship between different elements

- The grouping of input states (different rules referring to the same crisp values)

- Extensibility without compromising the original definition

- The possibility to provide a partial diagnostic output.

An ontology model-based representation provides the possibility to clearly define the relationships between diagnostic rules and inputs using axioms. Extensions to the diagnostic model are possible by introducing new concepts or axioms between concepts. The evaluation is also feasible by using an ontology reasoner. 
In order to provide partial diagnostic support, the usage of an inference system is required. The output of the desired inference system, would be made up of all the Minnesota code rules that have the required input at their disposal. For supporting partial diagnostic, the system needs to be reduced to its building blocks. These building blocks are used to create a customized diagnostic system, which can be evaluated based on the available input data. The relationship between the building blocks, diagnostic rules and inputs is defined by an ontology.

Ontology is a powerful knowledge representation formalism for modeling realworld concepts, basic mechanism and relationships used in different fields from semantic web modeling for the annotation of life events, goals, sub-goals, services, and other specific concepts from the public administration domain [7]. An ontology[2] (O) organizes domain knowledge in terms of concepts (C), properties $(\mathrm{P})$, relations $(\mathrm{R})$ and axioms $(\mathrm{A})$, and can be formally defined as a 4tuple $\mathrm{O}=(\mathrm{C}, \mathrm{P}, \mathrm{R}, \mathrm{A})$, where: $\mathrm{C}$ is a set of concepts defined for the domain. $\mathrm{A}$ concept is often considered as a class in ontology. $\mathrm{P}$ is a set of concept properties. A property $\mathrm{p} \in \mathrm{P}$ is defined as an instance of a ternary relation of the form $\mathrm{p}(\mathrm{c}, \mathrm{v}$, $\mathrm{f}$ ), where $\mathrm{c} \in \mathrm{C}$ is an ontology concept, $\mathrm{v}$ is a property value associated with $\mathrm{c}$ and $\mathrm{f}$ defines restriction facets on $\mathrm{v}$. $\mathrm{R}$ is a set of is a set of binary semantic relations defined between concepts in $\mathrm{C}$. $\mathrm{Rt}=$ \{one-to-one, one-to-many, many-to-many $\}$ is e set of relation type. A is a set of axioms. An axiom is a real fact or reasoning rule.

\section{Ontology-based Approach for the Minnesota Code}

\subsection{Analysis of the Minnesota Code System}

The diagnostic rules are the core and defining elements of the Minnesota code. This makes the diagnostic rules the starting point of analysis for constructing an ontology-based model. In order to provide an insight into how the diagnostic rules fit into the Minnesota code and provide the diagnostic process we will provide a detailed explanation for a specific use case. The "Q and QS Patterns" group will be used as a case study. This is the group for the rules that work with the Q and QS waveform patterns. This group can be further divided into three subgroups based on the ECG leads, meaning the Anterolateral site (I, aVL, V6), the Posterior site (II, III, aVF) and the Anterior site (V1, V2, V3, V4, V5). It can be observed that the same rule identification can occur under multiple subgroups. The difference in the rule occurrences is the ECG lead the diagnostic rules process. An example for this scenario is the rule 1-1-1. There are diagnostic rule definitions that are only present in the case of a specific sub-group (for example, the rule 1-13 is only present in the Anterolateral site [5]). 


\begin{tabular}{|c|c|c|}
\hline Rule Identifier & Group & Condition \\
\hline \multirow{7}{*}{ Rule 1-1-1 } & $\begin{array}{c}\text { Anterolateral site } \\
\text { (leads I, aVL, V6) }\end{array}$ & $\begin{array}{c}\text { Q/R amplitude ratio } \\
\geq 1 / 3, \text { plus Q } \\
\text { duration } \geq 0.03 \mathrm{sec} \\
\text { in lead I or V6. }\end{array}$ \\
\cline { 2 - 3 } & $\begin{array}{c}\text { Posterior (inferior) } \\
\text { site (leads II, III, } \\
\text { aVF) }\end{array}$ & $\begin{array}{c}\text { Q/R amplitude ratio } \\
\geq 1 / 3, \text { plus Q } \\
\text { duration } \geq 0.03 \text { sec } \\
\text { in lead II. }\end{array}$ \\
\cline { 2 - 3 } & $\begin{array}{c}\text { Anterior site (leads } \\
\text { V1, V2, V3, V4, V5) }\end{array}$ & $\begin{array}{c}\text { Q/R amplitude ratio } \\
\geq 1 / 3 \text { plus Q } \\
\text { duration } \geq 0.03 \text { sec } \\
\text { in any of leads V2, } \\
\text { V3, V4, V5. }\end{array}$ \\
\hline
\end{tabular}

Figure 2

The definition of the diagnostic rule 1-1-1

As seen in Figure 2, the definition of the diagnostic rule 1-1-1 is split into three parts. From the definition of the diagnostic rule it can be identified that two inputs are examined, the $\mathrm{Q} / \mathrm{R}$ amplitude ratio and the length of the $\mathrm{Q}$ waveform. In the diagnostic rule the input values are compared to predefined crisp values. In the case of the $\mathrm{Q} / \mathrm{R}$ amplitude ratio this would be greater than $1 / 3$, while in the case of the $\mathrm{Q}$ waveform length it would be greater than $0.03 \mathrm{~s}$.

The identification of the components that make up the system is the first step towards the construction of the ontology. In order to do this, the first step is to create a structured representation of the diagnostic rules. Representing the diagnostic rules as trees is one possible solution as shown in Figure 3. Figure 3 displays a generic tree representation of a Minnesota code diagnostic rule, where each diagnostic rule is an aggregation of predicate. The predicate branches represent the requirements as defined by a diagnostic rule. Each rule can contain one or more predicates. The predicate branch contains the ECG property that needs to fulfill the requirement as specified by the value node. A concrete example is shown in Figure 4, which is the tree structure based representation of the diagnostic rule 1-1-1 (original definition shown in Figure 2). The goal of this representation is to provide a method for identifying the core components and values of the Minnesota code. This is done through querying all ECG property nodes to identify the input types. Using the identified input nodes, one can query for all criteria values that belong to a specific input node type. The query results of the criteria values for a specific input property led to the conclusion that each input property is compared to a specific entry from a set of waveform states and never compared to a computed or dynamic value. As an example, let us consider 
the case of the $\mathrm{Q}$ waveform length, where the diagnostic rules examine various states, such as:

- Value that is in the range of $0.02 \mathrm{~s}$ and $0.03 \mathrm{~s}$

- Value that is in the range of $0.03 \mathrm{~s}$ and $0.04 \mathrm{~s}$

- Value that is greater than $0.04 \mathrm{~s}$

- Value that is greater than $0.05 \mathrm{~s}$

After conducting the same analysis for the other diagnostic rules belonging to the "Q and QS Pattern" group, it can be concluded that the Q waveform length has six possible states in the various rules. As the presented example shows, the studied parameter can be categorized into various states that have a strict definition. It can be concluded that the inputs, waveform states and diagnostic rules are the main concepts that make up the Minnesota code.

The Minnesota code does not define the waveform states. In order to gather all possible waveform states used in the diagnostic rules, an in-depth analysis of all diagnostic rules is needed. The in-depth analysis constitutes of the construction of the tree structure based representation for all diagnostic rules. Figure 3 provides a general overview of a tree structure representing a diagnostic rule which can have one or more predicates. The input types are represented by the ECG property nodes. By filtering out all unique instances we are able to acquire the input types. The possible waveform states are identifiable by paring the ECG property node occurrences (input types) with the value nodes (Figure 3 shows the relationship between the two node types). The diagnostic rules are clearly defined by the Minnesota code, coupled with the tree structure-based representation one has the necessary information for designing the ontology model.

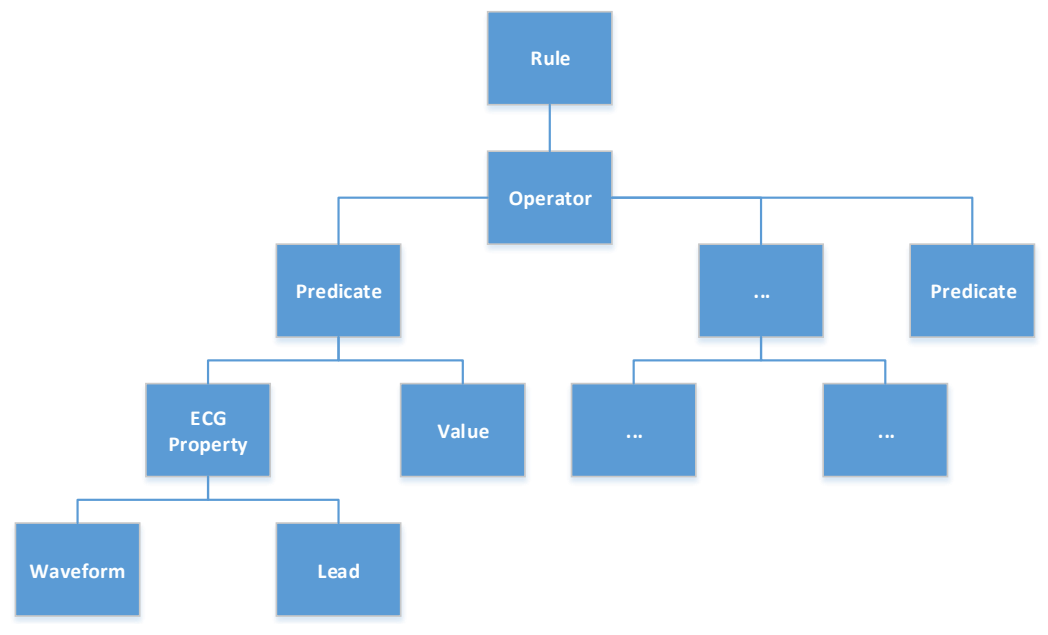

Figure 3

General tree structure based representation of diagnostic rules 


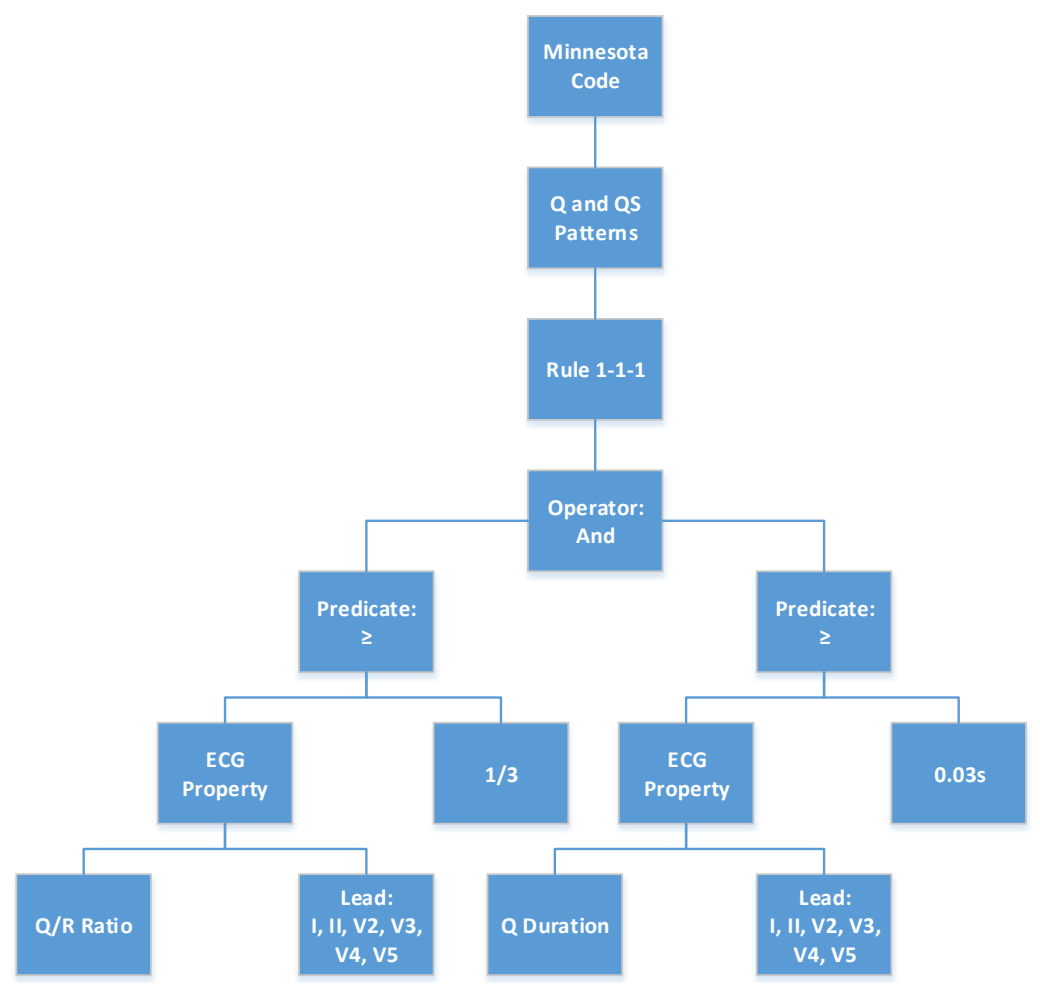

Figure 4

Tree structure-based definition of the Minnesota Code rule 1-1-1

\subsection{The Ontology Model}

The modeling of the ontology is done with the help of the Protégé [13] application. It is used for prototyping the ontology model and reviewing the results of the ontology inference systems. The inference system used by the authors is the Hermit reasoner [14].

The design of the ontology model starts with the identification of the building blocks of the Minnesota code. These building blocks correspond to the ontology concept representation of the elements identified by the analysis of Minnesota rules. In order to achieve a robust solution it is mandatory to have an ontology concept representation for even the smallest elements. The diagnostic inference is made possible by employing the correct relationships between these elements. Based on the original definitions, the elements used for constructing the rules are the waveforms, ECG leads, waveform value states and the grouping concepts. This means that the ontology model of the Minnesota code is divided into 4 main groups: Sample, ECG leads, Waveforms and Rules. 
The first group only contains a single concept, the "Sample". All input samples are an instance of the "Sample" concept. Various axioms are bound to the instances of the "Sample" concept to provide diagnostic inference support.

The second group is made up by the ECG leads and the corresponding instances. The ECG leads have 3 different sub-concepts (subclasses). These are the "AnteriorSite", "AnterolateralSite" and the "PosteriorSite". Since the ECG leads are globally used identifiers, each ECG lead group has a set of predefined instances for each ECG lead belonging to the specific sub-concept group. The ECG concepts and instances are used to describe the diagnostic rules in the ontology. Figure 5, shows the ontology model of the ECG leads and Figure 6, shows the "PosteriorSite" sub-concept definition in Protegé.

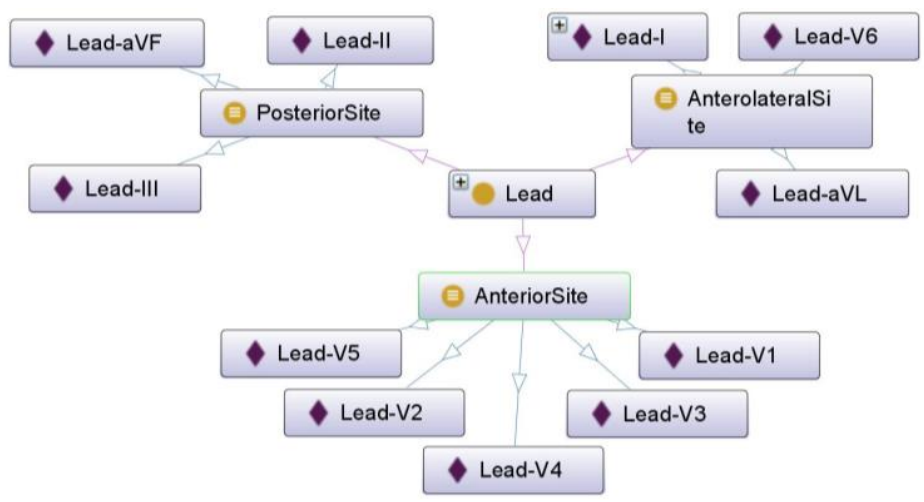

Figure 5

Graph of the Ontology model for the ECG leads created with Protégé [13]

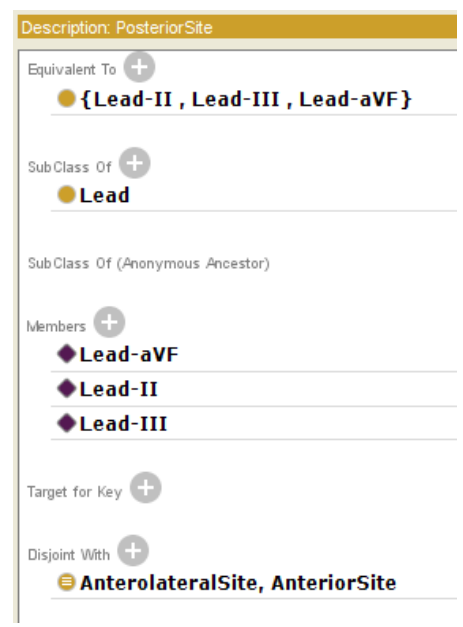

Figure 6

Ontology model of a specific lead group in Protegé 
The third major concept used in the ontology is the "Waveform" concept. As its name implies, this concept is used to represent the waveform characteristics. Every concept in the ontology that is a sub-concept of the "Waveform" concept, marks a parameter of the diagnostic system. The sub-concept tree is made up using the results of the conducted analysis of the Minnesota code rules. This means that all waveforms that were referenced at least once by one of the diagnostic rules. The "Waveform" concept has approximately 17 sub-concepts. The major waveforms concepts are shown in Figure 7. The sub-concepts of these groups contain the state concepts for each waveform. The ontology concepts representing the specific waveform states have been designed based on the analysis results of the rules. Each waveform state concept uniquely identifies a waveform type and crisp value/range found in one of the Minnesota code diagnostic rules. The state concepts are unique to the waveform type that they belong to. A different waveform type can have a state that matches the same crisp state. In the ontology model, it is important to differentiate between different waveform types regardless of their crisp states, in order to support the inference system.

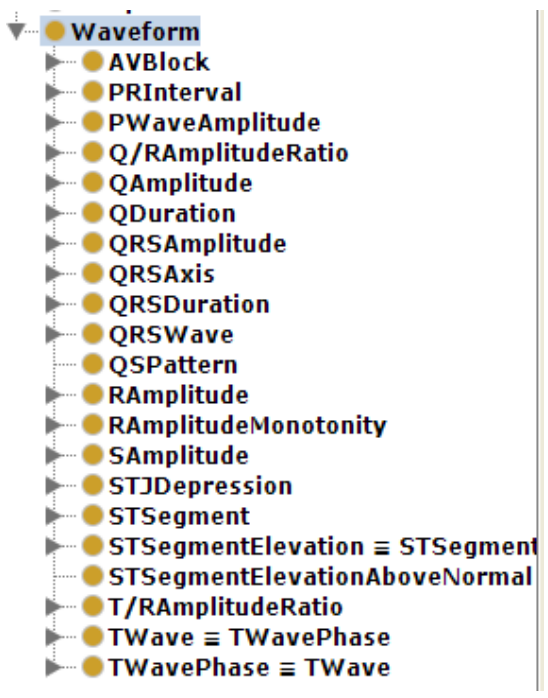

Figure 7

Waveform groups

The major functionality of the waveform grouping concepts is to identify the waveform type of a specific waveform state provides based on the original Minnesota code definitions. Every waveform grouping concept contains one or more possible state concepts. Figure 8, shows the Q waveform length ontology based state concepts. A distinctive property of the waveform state concepts is a data attribute that captures their state value. For example, the "QDurationLong" concept defines that the value of the instance is greater than or equal to $0.05 \mathrm{~ms}$. 


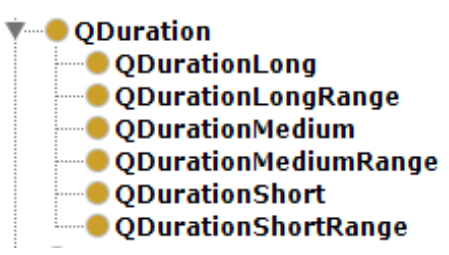

Figure 8

Ontology model of the Q waveform state

The fourth and last major group contains the ontology representation of the diagnostic rules, which are all sub-class (sub-concepts) of the "Rule" concept. The direct descendants correspond to the rule groups defined by the Minnesota code. The diagnostic rules defined by the Minnesota code are modeled as equivalent classes in the ontology (Figure 9). This means that the instances for these concepts are identified based on a specific set of properties/attributes being present for an ontology instance. Diagnostic rule instances are not created or classified directly in the ontology model. This approach is relevant for establishing the diagnostic output. In ontologies, compared to regular classes (concepts) equivalent classes represent a two-way relationship. What this means in practice is that if an ontology instance has the necessary properties, it can be categorized as an instance of an equivalence class, regardless of the existing and established hierarchical relationship. Every diagnostic rule is modeled as a single equivalence class, i.e. a one-to-one mapping compared to the original Minnesota rules. In a scenario, where a set of requirements defined by one of the equivalence classes are met by an instance, the ontology will mark the instance as a type of that specific diagnostic rule class. This method is applied for identifying all the diagnostic rules applicable for a specific ECG sample. Figure 9, shows the structure of diagnostic rule 1-1-1 in the ontology model. The representation is close to the original definitions. The difference is in the application of concepts to represent waveform states. The ontology representation could have applied the same approach, where rules would refer to crisp values. This approach was abandoned in favor of concepts providing the possibility to use various representations for waveform values (fuzzy, crisp, interval-based etc.). In the ontology, every measured value is an instance of a concept. The waveform instances can have various axioms in addition to the measured value. For example, one can extend a waveform instance to have a fuzzy definition.

(hasLead some ( $\{$ Lead-I , Lead-II , Lead-V2 , Lead-V3 , Lead-V4, Lead-V5, Lead-V6\})) and (hasWaveform some Q/RAmplitudeRatioUpperTreshold) and (hasWaveform some QDurationshort)

Figure 9

Ontology representation of Minnesota code diagnostic rule 1-1-1

Inside the ontology the various types, properties and values are connected together with axioms. In the case of the diagnostic system, a predefined set of axioms is 
used. For example, an axiom is used to connect the measurement value to the "Waveform" concept instance. This axiom is a function that operates on "Waveform" concepts and floating point values. The described axiom is called "hasCrispValue". The exact functionality of the "hasCrispValue" is to define the measurement/input value of an ECG waveform. The "hasCrispValue" and similar axioms such as "hasWaveform", "hasLead" are used by the rule equivalence class definitions. These axioms operate on a specific ontology instances and connect the various ECG properties to that instance. In the case of the "hasWaveform" axiom, the operands always have the type of Waveform and Sample, while the "hasLead" axiom operates on a Waveform instance and a Lead instance.

\section{Ontology-based Diagnostics}

The first step towards providing a diagnostic output is the population of the Minnesota code ontology system. One by one, all the available ECG samples are added to the ontology. For every sample, the same algorithm is executed. The first step of the algorithm is to create the ontology representation for the input, meaning an instance from the "Sample" concept. After creating the instance, the algorithm sets the known properties. The algorithm does this by creating the necessary connections in the ontology, using various axioms ("hasLead", "hasWaveform", "hasCrispValue"). ECG leads and the corresponding waveform values are attached to the ontology instance. Since axioms operate on ontology instances, the available waveform properties need to be created. Inputs belong to a specific "Waveform" concept. Waveforms are grouped by type, all groups contain the concepts representing the specific states for a particular waveform. To find the appropriate waveform type concept for an input value, the diagnostic system uses an inference system to acquire all the specific sub-concepts (waveform states) for a waveform type. Figure 10 illustrates a result of the ontology populating step in Protegé. The Figure displays an instance of "Sample" called "testSample" and the corresponding axioms for the "Waveform" properties belonging to the "testSample".

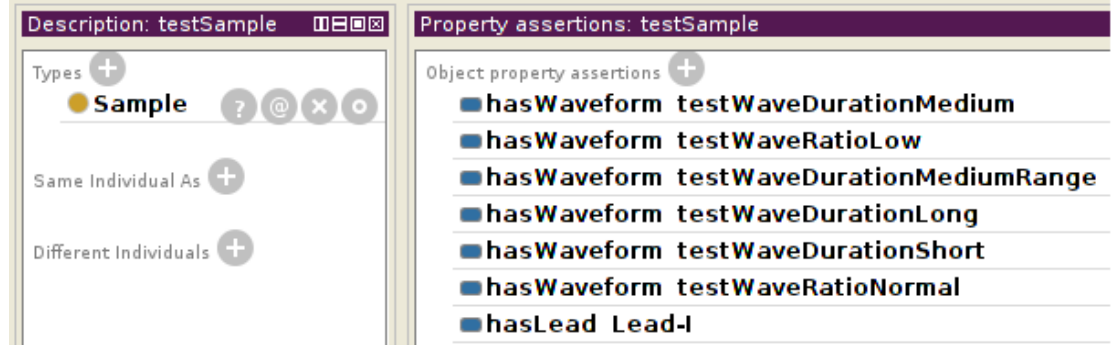

Figure 10

Definition of a "Sample" instance and the corresponding waveforms in Protegé 
The result of the inference system is a list of concepts. For all the acquired concepts, the diagnostic algorithm creates an instance with the type and measured value set. The same measured value is set for all the state instances. As an example, a case study of $\mathrm{Q}$ waveform length is conducted. The $\mathrm{Q}$ waveform length is used by the Minnesota code. In the case of this waveform type (Q) and its parameter (length), the Minnesota code differentiates between 6 states (sets). The ontology system handles the mapping for the specific states. This step is required since in the original Minnesota code rules this waveform property is represented as a single factor, while in the ontology-based solution it is divided into 6 factors because of the fact that the ontology model works with waveform states. For all 6 states the same measured value is set, the difference is in the ontology types of the instances.

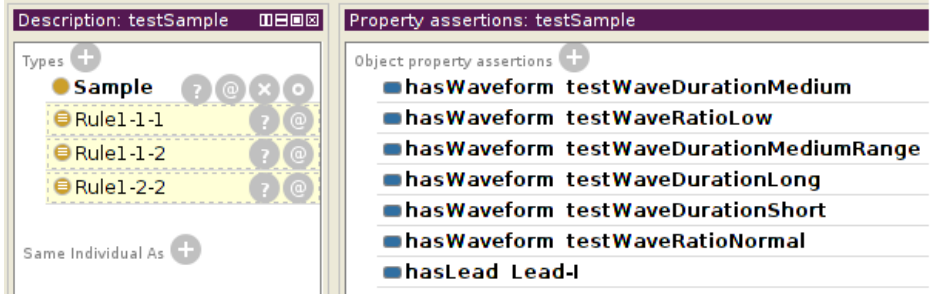

Figure 11

Definition of a "Sample" instance and the corresponding waveforms in Protegé after the rule inference step

After the ontology is populated with the available information, the next step is to identify which diagnostic rules have the required information available for evaluation. In the ontology the definition of diagnostic rules is performed with equivalent classes. This implies that the input samples inferred types contain all the diagnostic rule types that can be evaluated. The type - diagnostic rule identification is performed by an ontology reasoner [14]. Figure 11 displays the result produced by the Hermit [14] reasoner for the "testSample" shown in Figure 10. As the picture shows, the reasoner identified that "testSample" meets the criteria for 3 possible "Rule" sub-concepts.

After the inference system finished the execution, the types of the sample instance need to be analyzed. All the type associations of the sample instance that have the parent class of 'Rule' mark the diagnostic rule types that have all the required information available for evaluation. The evaluation step starts with acquiring all the types associated with the ECG sample that are the sub-types of the diagnostic rule modeling concept. In the case when the number of diagnostic rule representing types is greater than zero, all the diagnostic rules are evaluated. Since all the waveform concepts have a crisp value associated through the "hasWaveformValue" axiom, the evaluation is a matter of logical comparison. The truthiness values of the waveforms need to be aggregated into a single value thus producing the diagnostic output for a specific rule. 


\section{Conclusions}

Our ontology-based Minnesota code model meets the outlined goals. It models the basic concepts of the diagnostic system and the related relationships. The input types and states are explicitly defined. Using the explicit definitions partial diagnosis is also supported using a two-step approach. First, it is determined which diagnostic rules have all the mandatory information available, then the identified rules are evaluated. The compatibility between the original expert system based definitions and the ontology based solution is ensured by crosschecking the results of the ontology model based solution with the results produced by the expert system based solution. By default, both solution use the same methodologies. However, the ontology based definitions are based on state concepts instead of value ranges. The state concepts are the key to the extensibility of the system. Besides the Minnesota code-based value range definitions it is possible to attach various other representations and evaluation algorithms to apply different diagnostic algorithms as well. For example, all waveform states can have fuzzy definitions associated with them. In most fuzzyontology solutions the fuzzification of the ontology concepts is carried out using ontology annotations, when a concept has associated multiple fuzzy membership functions. Using this approach, all the ontology concepts represent a single fuzzy variable, where the fuzzy membership functions are stored in an ontology annotation. In the case of the Minnesota code ontology model, the state concepts can be used to store the membership function definitions. For each state concept one membership function definition is assigned. The outcome of this approach is that a fuzzy variable is represented by a grouping concept and the sub-concepts contain the membership function definitions of the variable. The task of the ontology system becomes identification of the branches of the decision tree, which can be evaluated. Usage of the fuzzy characteristics is required for the evaluation of the diagnostic rules. The application of fuzzy logic, in the diagnostic steps, through the usage of fuzzy-ontology, is the next step for improving the current solution presented herein.

\section{Acknowledgement}

The research was supported by the Hungarian OTKA projects 106392 and 105846, and project of the Vojvodina Academy of Sciences and Arts "Mathematical models of intelligent systems and theirs applications".

\section{References}

[1] Peter W. M, Shahid L., "Automated Serial ECG Comparison Based on the Minnesota Code", Journal of Electrocardiology, Vol. 29, Sup. 1, pp. 29-34, 1996

[2] G. Balázs, K. Haraszti, Gy. Kozmann, "Increasing the Efficiency of the "Excluding Rules" of the Minnesota Coding System using the Fuzzy Logic", Measurement Science Review, Volume 5, Section 2, 2005 
[3] Fernando Bobillo, Umberto Straccia, "Fuzzy Ontology Re Presentation using OWL 2", International Journal of Approximate Reasoning, 2011, pp. 1073-1094

[4] Sram, N., Takacs, M. "Minnesota Code: A Fuzzy Logic-based Approach", Proc. of the $11^{\text {th }}$ International Symposium on Computational Intelligence and Informatics (CINTI 2010), pp. 233-236, Budapest, Hungary, 2010

[5] Prineas, Ronald J., Crow, Richard S., Zhang, Zhu-ming, "The Minnesota Code Manual of Electrocardiographic Findings", ISBN 978-1-84882-777-6

[6] Engelstein ED, Zipes DP., "Sudden Cardiac Death", The Heart, Arteries and Veins. New York, NY: McGraw-Hill; 1998:1081-1112.4

[7] Myerburg RJ, Castellanos A., "Cardiac Arrest and Sudden Death", Heart Disease: A Textbook of Cardiovascular Medicine. Philadelphia, Pa: WB Saunders; 1997:742-779

[8] de Vreede Swagemakers JJM, Gorgels APM, Dubois-Arbouw WI, van Ree JW, Daemen MJAP, Houben LGE, Wellens HJJ. "Out-of-Hospital Cardiac Arrest in the 1990's: a Population-based Study in the Maastricht Area on Incidence, Characteristics and Survival", J Am Coll Cardiol. 1997; 30:1500-1505

[9] World Health Organization, "Annex Table 2: Deaths by Cause, Sex and Mortality Stratum in WHO Regions, Estimates for 2002", The world health report, 2004

[10] Sándor Khoór, István Kecskés, Ilona Kovács, Dániel Verner, Arnold Remete, Péter Jankovich, Rudolf Bartus, Nándor Stanko, Norbert Schramm, Michael Domijan, Erika Domijan, "Heart Rate Analysis and Telemedicine: New Concepts \& Maths", Proceedings of the IEEE SISY 2007, pp. 61-66, Subotica, Serbia, 2007

[11] Ceusters W Ab, Smith B Bcd, De Moor G A, "Ontology-based Integration of Medical Coding Systems and Electronic Patient Records", IFOMIS Reports, 2004

[12] John R. Hampton, “The ECG Made Easy $8^{\text {th }}$ Edition”, 2013

[13] http://protege.stanford.edu

[14] Birte Glimm, Ian Horrocks, Boris Motik, Giorgos Stoilos, Zhe Wang, "HermiT: An OWL 2 Reasoner", Journal of Automated Reasoning, October 2014, Volume 53, Issue 3, pp. 245-269

[15] Goldberger AL, Amaral LAN, Glass L, Hausdorff JM, Ivanov PCh, Mark RG, Mietus JE, Moody GB, Peng C-K, Stanley HE. PhysioBank, PhysioToolkit, and PhysioNet: Components of a New Research Resource for Complex Physiologic Signals. Circulation 101(23):e215-e220 [Circulation Electronic Pages; http://circ.ahajournals.org/cgi/content/full/ 101/23/e215]; 2000 (June 13)

[16] Doina Drăgulescu, Adriana Albu, "Medical Predictions System", Acta Polytechnica Hungarica Vol. 4, No. 3, 2007, p. 89 